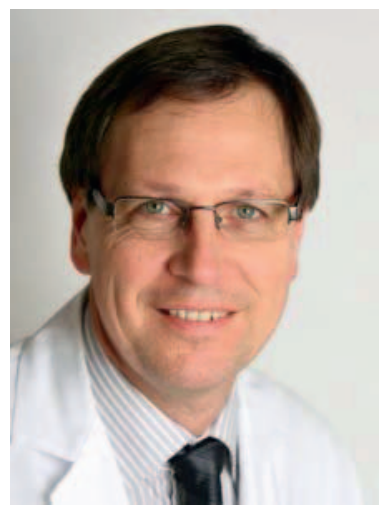

\title{
Integrative Onkologie - «... sich Zeit nehmen und den Krebspatienten menschlich begegnen ....»
}

\section{Interview mit Prof. Dr. med. Marcus Schuermann}

Die Diagnose «Krebs» bedeutet heute nicht mehr automatisch den sicheren Tod. Selbst bei bösartigen Erkrankungen wie dem malignen Melanom im fortgeschrittenen Stadium stehen heute erste wirksame Medikamente zur Verfügung. Die Frühentdeckung von Tumoren gelingt häufiger dank besserer bildgebender Verfahren wie auch dank der Tumorchirurgie, die sich qualitativ erheblich verbessert hat. Entsprechend ist auch die Sterblichkeit an Krebs in der Schweiz rückläufig, wobei hier allerdings auch die Prävention, wie z.B. das veränderte Raucherverhalten, eine Rolle spielt. Prof. Dr. med. Marcus Schuermann (Abb. 1, 2), Leiter der Onkologie an der Aeskulap-Klinik in Brunnen, gibt im Interview mit Dr. Josef Roos Auskunft über den Stand der Onkologie im Allgemeinen und seine Integrative Onkologie im Speziellen.

In welcher Weise hat sich in den letzten Jahrzehnten die Lebenserwartung bei Krebs verändert?

Die durchschnittliche Lebenserwartung für die folgenden 5 Jahre beträgt derzeit im Durchschnitt aller Tumorerkrankungen um die 60-65\%; die Mehrheit verdankt dies natürlich der verbesserten Diagnostik und der besseren Chirurgie. Aber auch bei unheilbaren Erkrankungen ist die Behandlungszeit wesentlich verlängert worden. Am Beispiel des Darmkrebses wird deutlich, was die letzten 40 Jahre ergeben haben. Um 1970 1980 herum lag die mittlere Überlebenszeit noch bei 9-12 Monaten unter einer einfachen Chemotherapie; heute erreichen wir mit aufeinanderfolgenden Kombinationstherapien und Hinzunahme von monoklonalen Antikörpern (immunologisch aktive Antikörper) um die 24-27 Monate.
Wo steht heute die Krebsforschung gegenüber den 1980er-Jahren?

In den 1980er-Jahren haben die Molekularbiologie und Molekulare Genetik die Grundlagenwissenschaft revolutioniert. Dazu wurde es über Zellfusion möglich, auch Antikörper klonal herzustellen. Heute profitieren wir von neuen zielgerichteten $\mathrm{Me}$ dikamenten, die etwa 15 Jahre verspätet Einzug in die Klinik halten. Auf Forschungsebene ist derzeit die systematische Genomsequenzierung von Tumoren spannend, da es sich herausstellt, dass immer wieder bestimmte genetische Veränderungen als die Hauptverantwortlichen für die Entstehung von Krebs benannt werden können. Die Zahl dieser Orte ist begrenzt. Es handelt sich um die ca. 100 Schlüsselstellen, deren Genprodukte in gut einem Dutzend Signalwege eine entscheidende Rolle spielen. Theoretisch sollte es möglich sein, Medikamente zu entwickeln, die allein oder in vielfältiger Kombi-

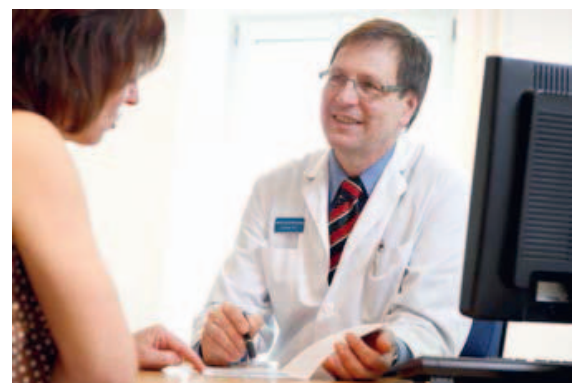

Abb. 1. Prof. Dr. med. Marcus Schuermann im Patientengespräch.

nation solche gestörten Signalwege in der Zelle neutralisieren könnten. Auch die Immunologie, d.h. die Wissenschaft um das Funktionieren unserer Immunabwehr, insbesondere in Hinblick auf Tumorabwehr, macht grosse Fortschritte. Zunehmende Erkenntnisse in der Feinregulation helfen, erste Medikamente zu entwickeln, die ein besseres Erkennen und eine effektivere Bekämpfung von Tumorzellen erlauben. Natürlich ist es noch ein Weg bis zum funktionierenden Medikament.

\section{KARGER}

Fax +49761 4520714 Information@Karger.de Information@Kar
(๑) 2012 S. Karger GmbH, Freiburg
Dr. Josef Roos

Medienverantwortlicher Aeskulap-Klinik

Gersauerstrasse 8, 6440 Brunnen, Schweiz

josef.roos@aeskulap.com 


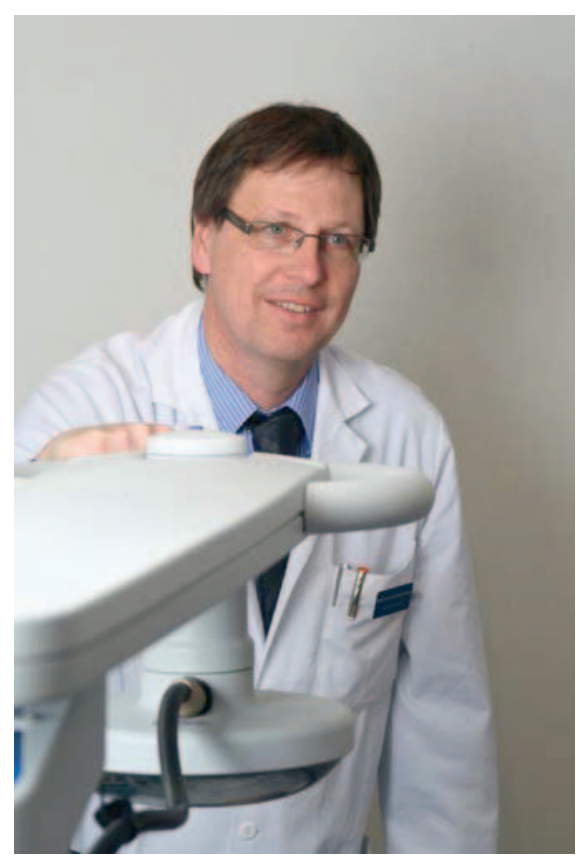

Abb. 2. Prof. Dr. med. Marcus Schuermann bei der Behandlung mit dem Hyperthermiegerät der Aeskulap-Klinik.

Wie sieht das Nebenwirkungspotenzial der konventionellen onkologischen Behandlung aus?

Im Grossen und Ganzen sind die meisten Therapien mit wenigen Ausnahmen weiterhin nebenwirkungsreich. In der Praxis spielt die Therapiegestaltung eine wichtige Rolle. Insofern sollten der Onkologe und seine Erfahrung eine grosse Rolle spielen, anders vielleicht noch als vor 10 Jahren.

Sie sagen, dass sich die Kombination traditionelle Behandlung gekoppelt mit Komplementärmedizin in sich beisst. Weshalb?

Das stimmt nicht unbedingt. Klar ist, dass die Mehrzahl komplementärer Verfahren kein Ersatz für eine Tumortherapie ist, hingegen auf der Seite der Stützung des Körpers sehr willkommen sein können. Hier ist es wichtig, dass man bei naturheilkundlichen Medikamenten die Inhaltsstoffe kennt, die unter Umständen mit konventionellen Krebsmedikamenten in Wechselwirkung treten können. Hier hilft oft eine Pause für wenige Tage oder ein zeitversetztes Einsetzen, um dieses Risiko zu vermeiden.

Sie haben eine natürliche Vorgehensweise entwickelt. In der AeskulapKlinik wenden Sie die sogenannte Integrative Krebstherapie an. Was steckt dahinter?

Grundsätzlich nutzen wir alle Möglichkeiten in der Krebstherapie, die wir zur Verfügung haben. Wir haben generell noch eher zu wenig als zu viel Auswahl. Wenn wir aber vorurteilsfrei alle klassischen wie komplementären Verfahren nutzen, führt das automatisch zu mehr Angebot. Komplementäre Verfahren nutzen wir eher in der Stützung, klassische Verfahren eher in der Therapie.

Wo wird Ihre Integrative Onkologie in der Schweiz sonst noch angewendet?

Es gibt mittlerweile mehrere Orte, wo komplementärmedizinische Verfahren im Klinikrahmen angewendet werden. Allen voran sind drei anthroposophisch orientierte Kliniken zu nennen, die dies schon seit Jahren tun. Am Kantonsspital St. Gallen wird nunmehr neu ebenfalls ein Zentrum für Integrative Onkologie in Flawil eröffnet. Im universitären Rahmen gibt es an der Universität Zürich eine ambulante Sprechstunde am Institut für Naturheilkunde wie auch in Bern an der KIKOM (Kollegiale Instanz für Komplementärmedizin). Komplementärmedizinische Verfahren in der Behandlung onkologischer Patienten werden ebenfalls an der ParacelsusKlinik Lustmühle in Teufen wie auch an der Clinica Santa Croce in Orselina angeboten.
Welche Methoden wenden die reinen Schulmediziner an? Und wo sehen Sie dabei Probleme für den Patienten?

Heute wird bei jeder Tumorentfernung zunächst geprüft, welche Eigenschaften ein Tumor in Hinblick auf genetische Veränderungen oder die Ausprägung bestimmter Eiweissstoffe hat. Dann erfolgt die Abstimmung auf den Einsatz von vorhandenen zielgerichteten Medikamenten. Neben zahlreichen Zytostatika, die heute weitgehend noch immer im Einsatz sind, gibt es die Antikörperbehandlung allein oder in Kombination mit Zytostatika, dazu mittlerweile auch zielgerichtete Medikamente in Tablettenform, die speziell in Signalwege von Tumorzellen eingreifen. Ein Nachteil der modernen Medikamente ist, dass sie trotz verbesserter Wirkungsweise teilweise doch erhebliche Nebenwirkungen haben, die denen der Chemotherapie bisweilen gleichkommen. Insbesondere sehen wir das gefürchtete Müdigkeitssyndrom, Appetitlosigkeit und Magen-DarmStörungen doch häufiger, als uns lieb ist. Einige Medikamente haben uns auch mit völlig neuen Nebenwirkungen konfrontiert, deren Behandlung eine Herausforderung ist, insbesondere bei ungewohnten Hautreaktionen.

Weshalb überfordert die Therapie von Krebserkrankungen die meisten Patienten?

Eine medikamentöse Krebstherapie ist heute eine immer noch einschneidende Therapie. Viele Patienten sind nicht hinreichend über die Nebenwirkungen von Medikamenten informiert. Und wie sie sich besser vor Risiken und Komplikationen schützen können. Hier fehlt oft die Zeit in den Ambulatorien. Der Patient versteht meist in Sorge um das Bild seiner Erkrankung nicht alles, was in der Sprechstunde gesagt wurde. Es 
ist in diesem Rahmen wichtig, sich viel Zeit zu nehmen und Dinge zu wiederholen. Auch denke ich, dass die Mehrzahl der Therapien an den Patienten anzupassen ist, als dass man an Schemata festhält. Zur Abwägung alternativer Möglichkeiten sollte meiner Meinung nach zur Therapiefestlegung auch eine Zweitmeinung eingeholt werden. Mindestens dann, wenn Unklarheiten bestehen.

«Maximale Wirkung bei minimalen, vertretbaren Nebenwirkungen» lautet Ihre Maxime. Welche Ansatzpunkte sind dabei integraler Bestandteil?

Dies erfordert ein ganzheitliches Assessment mit leider sehr zahlreichen Einzelaspekten. Einflussgrössen in Hinblick auf die Therapieentscheidung sind: Alter und Gesundheitszustand, Begleiterkrankungen, Krebsartund Ausbreitungsstand, Abstufung der vorhandenen Therapiemöglichkeiten, Nebenwirkungen dieser Möglichkeiten, Behandlungsmöglichkeit der Nebenwirkungen mittels Komplementärmedizin, der persönliche Behandlungswunsch, der soziale Hintergrund (eingebettet sein). Zuletzt spielt bei Wahlleistungen auch der Zusatzversicherungsrahmen bzw. der eigene finanzielle Hintergrund eine Rolle.

Stützung des Körpers - was bedeutet das und wie gehen Sie hier vor?

Hier haben sich wenige Massnahmen als sehr effektiv erwiesen: In der Akutphase gravierender Nebenwirkungen geben wir aufbauend hochdosierte Vitamin-C-Infusionen und bisweilen Thymuspeptide oder eine Ozontherapie. Während einer fortlaufenden Chemotherapie sehen wir den Patienten meist einmal in der Woche zur ausführlichen Besprechung seines aktuellen Zustandes.
Basisprogramm sind auch hier Vitamin-C-Infusionen, eine Immunmodulation mittels Mistel sowie eine Atemausdauertherapie und ausführliche Anleitung zu körperlicher Bewegung im Alltag. Ein psychologisches Coaching, eine Ernährungsberatung und eine Schmerztherapie stehen mit im Programm, werden aber nur da, wo notwendig bzw. gewünscht, umgesetzt. Natürlich widmen wir uns auch den einzelnen Nebenwirkungen so gut wir können - mit konventionellen wie auch naturheilkundlichen Verfahren.

Der Mensch als Gesamtheit steht bei Ihnen im Mittelpunkt der Therapie. Wie zeigt sich das?

Sich ausführlich Zeit nehmen, menschliche Begegnung, Besprechen aller konventionellen Möglichkeiten wie auch der komplementärmedizinischen Begleitung. Abstimmen eines ganzheitlichen Programms auch mit Blick auf Erhaltung der Therapiefähigkeit in der Zukunft (Nachhaltigkeit). Sicherstellen, dass alles verstanden ist.

Was verstehen Sie unter Ihrer onkologischen Begleitung? Was umfasst diese Vorgehensweise?

Die onkologische Begleitung dient dem Ziel, schon während der Bestrahlung oder Chemotherapie allzu grosse Nebenwirkungen, die die Lebensqualität einschränken, zu vermeiden oder zu vermindern. Es gilt hier das Prinzip: Je frühzeitiger, desto besser. Mit der onkologischen Begleitung verfolgen wir einen Langzeitansatz, wobei der Patient meist einmal wöchentlich ambulant für einen halben Tag die Klinik aufsucht. Zum Einsatz kommen sowohl schul- als auch komplementärmedizinische Verfahren, die vorwiegend stützend wirken und die Regeneration beschleunigen.
Ziel des Programms ist eine Bekämpfung des oft gefürchteten Müdigkeitssyndroms, eine Intensivierung der Abwehrkräfte und vor allem Motivation für die Weiterführung der Therapie.

\section{Welche Schwerpunkte setzen Sie?}

Die integrative Behandlung besteht aus folgenden Schwerpunkten:

- Onkologische Behandlung: Gemäss den aktuellen Leitlinien der onkologischen Fachgesellschaften eine der Krebsart, dem Ausbreitungszustand und dem Patienten gerechte medikamentöse Therapie, im Einzelfall auch individuell an seine Lebensvorstellung angepasst.

- Zusätzliche onkologische Therapieangebote: i) lokoregionale Tiefenhyperthermie bei Tumoren in Begleitung $\mathrm{zu}$ konventionellen Therapien; ii) spezielles Phytotherapieangebot beim lokalen und fortgeschrittenen Prostatakarzinom.

- Onkologische Begleitung: In diesem Programm widmen wir uns allen Aspekten der Krebserkrankungen ausserhalb der eigentlichen Krebstherapie, also der körperlichen und seelischen Stützung auf vielfältige Weise, sowie vielen Informationsfragen rund um das Thema Krebs.

Welchen zeitlichen Aufwand muss ein Patient dafür einsetzen?

Er sollte mindestens einmal in der Woche für einen halben Tag nach Brunnen kommen können. Die Hyperthermie erfolgt in der Regel zweimal wöchentlich. Bei weniger intensiven Therapien reicht bisweilen einmal monatlich.

Welche Rolle spielt hier der psychologische Aspekt? 
Die psychologische Begleitung, wir nennen es "Coaching», vermittelt dem Patienten über die Wahrnehmung seiner Erkrankung und gleichzeitige Besinnung auf seine Bedürfnisse eine Wiedererlangung seiner persönlichen Souveränität als handelnde Person. Die äusseren Einflüsse der Diagnose und die nachfolgenden gravierenden Behandlungen lösen starke Ängste aus, die häufig auch einen sozialen Rückzug nach sich ziehen. Hier braucht es häufig professionellen Rat, sich dieser isolierenden und zum Teil selbstaufgebenden Haltung zu entziehen und dem Leben auch in seinen Einschränkungen wieder mehr Raum zu geben.

Welche Vorteile sehen Siegrundsätzlich bei Ihrer Integrativen Krebstherapie?

Es ist kein Geheimnis, dass aufgeklärte und sich ihrer Erkrankung widmende Patienten einen erheblichen Überlebensvorteil aufweisen. Integrative Onkologie hilft hier in der ganzheitlichen Wahrnehmung und Bewältigung, der situationsgerechten Therapieanpassung in der Stützung des Körpers schon während der Therapien und letztlich auch in der Informationsvermittlung über alle Verfahren der Behandlung, der Nebenwirkungen und dem vorausschauenden Begegnen von möglichen Komplikationen.

Weshalb lässt sich hier die Kombination Schulmedizin und Komplementärmedizin miteinander vereinbaren?

Voraussetzung für eine sinnvolle Kombination von klassischen und naturheilkundlichen Therapien ist die genaue Kenntnis der einzusetzenden klassischen Medikamententherapie sowie der naturheilkundlichen Verfahren. Diese muss der Facharzt kennen, um dem Laien die Chancen auf Besserung, aber auch die im Einzelfall möglichen Risiken einer Interaktion aufzuzeigen. Fakt ist, dass wir durch die Kombination eine reiche Palette von Therapieangeboten haben, die sich besser auf die Individualität und die Bedürfnisse des Patienten ausrichten als ein rein schulmedizinisches Angebot. Dies, ohne dass wir auf die Wirksamkeit der Therapie insgesamt verzichten müssen. Wir achten sehr auf zeitlich abgestimmte Therapien, um eine gegenseitige Behinderung zu vermeiden. Darüber hinaus haben wir ein Gespür, wo vielleicht eine komplementärmedizinische Vorgehensweise reicht und wo vielleicht bei drohender Gefahr doch eher klassische Verfahren angesagt sind.

Die Aeskulap-Klinik verfügt für die Krebstherapie auch über ein hochmodernes Hyperthermiegerät. Was bewirkt dieses Gerät?

Eine Einsatzmöglichkeit besteht im Prinzip bei allen lokalisierten, aber nicht mehr heilbaren Krebsarten. Besonders geeignet, d.h. thermoempfindlich, sind die Weichteilsarkome und Mesotheliome, aber auch Tumoren im Hals-, Thorax- und Beckengebiet, da hier die lokale Hitzeausbreitung im Tumorgewebe optimaler läuft. In den 3 Jahren der Anwendung unseres neuen Gerätes haben wir über 2500 Therapien durchgeführt. Die dabei gewonnene Erfahrung hilft uns bei der Therapieeinschätzung. Nebenwirkungen treten dabei glücklicherweise selten auf.

Bei Ihnen gibt es auch Palliative Care. Wie sieht die aus?

Palliativmedizin ist für sich eigentlich immer eine ganzheitliche Medizin. Insofern ist die Behandlung am Lebensende für uns natürlich. Den Satz «Für Sie können wir leider nichts mehr tun» gibt es bei uns nicht, es gibt immer etwas zu tun - auch in den letzten Tagen und Stunden. Wir sind aktiv beteiligt im kantonalen Konzept für Palliative Care und haben ein in der Palliativmedizin geschultes Personal, das diese Medizin - sei es ambulant oder stationär - auch gerne ausführt. Unser Haus ist auch von seiner bevorzugten Lage am See, von seiner Infrastruktur wie auch von seiner hotelartigen Erscheinungsform her generell ein bevorzugter Platz für Palliative Care. Natürlich tragen wir dieses Anliegen auch im Herzen.

Wie sieht die Akzeptanz Ihrer Vorgehensweise bei den reinen Schulmedizinern aus?

Es findet eine zunehmende Vernetzung wie auch ein Austausch statt. Viele Kollegen haben begriffen, dass in der Aeskulap-Klinik im onkologischen Bereich primär onkologisch gedacht wird und man mit ihnen in fachlicher Hinsicht eine Sprache spricht. Das hilft in der Akzeptanz und nimmt den Kollegen die Angst.

Wo will sich die Aeskulap-Klinik gesamtschweizerisch in der Onkologie positionieren?

Wir sind Mitglied in fachlich überregionalen Verbänden im Zeichen der Qualitätssicherung - einerseits in onkologischer Hinsicht als Mitglied im Swiss Cancer Network. Hier wird die Einhaltung von hohen Standards sichergestellt. Damit verfügt der Paient über einen zertifizierten Onkologen und Zugang zu einer optimalen, fachübergreifenden Behandlung und Betreuung, die den aktuellen Qualitätsstandards der Fachgesellschaften entspricht. Andererseits soll über den Austausch und die Angleichung komplementärmedizinischer Verfahren in einem europäischen Verbund sichergestellt werden, dass 
effektive naturheilkundliche Verfahren und keine Aussenseitermethoden angewandt werden. Die Aeskulap-Klinik ist daher auch aktives Mitglied in der ESIO (European Society for Integrative Oncology). In jedem Fall wird die Aeskulap-Klinik immereine innovative und über die konventionellen Möglichkeiten und Standards hinausdenkende Klinik sein, die für einen Rat suchenden Patienten von Interesse ist.

Sie empfehlen ausdrücklich, eine Zweitmeinung einzuholen. Weshalb?

Es ist heute generell so, dass die Diagnose Krebs in einem Rahmen erfolgt, in dem viele Arzt- und Untersuchungstermine in medizinischen Zentren stattfinden. Dies ist generell zwar in Hinblick auf die Qualitätssicherung der diagnostischen Einschätzung sinnvoll, erlaubt aber dem Betroffenen nicht, in Ruhe und unabhängig von Interessen aller Beteiligten eine neutrale Einschätzung zu erlangen. Zudem ist die eigene seelische Anspannung zu Beginn so hoch, dass nur ein Bruchteil verstanden wird. Ein «Empowerment» des Patienten ist aber heute für alle weitreichenden Entscheidungen erwünscht. Insofern braucht es einen Ort, an dem ein unbeteiligter, aber onkologisch erfahrener Arzt einmal unter ganzheitlichen Gesichtspunkten alle Möglichkeiten durchspricht und bei der Therapieentscheidung hilft.

Interview: Josef Roos 